\title{
Bibliotheken sind "smart « geworden Ein Plädoyer für ein bewusstes »smart« machen in den nächsten Jahren
}

Frank Gillert

Vor mehr als zehn Jahren haben RFID-Systeme Einzug in die deutsche Bibliothekswelt gehalten. Heute kann konstatiert werden, dass rund 1000 öffentliche und wissenschaftliche Einrichtungen damit ausgestattet und rund 20 Millionen Medien »smart geworden « sind. Der folgende Beitrag soll auf Basis des Innovations- und Technologiemanagements aufzeigen, welche Mechanismen zu dieser Entwicklung geführt haben und welche Erkenntnisse für eine weitere, bedarfsorientierte Technologisierung des Bibliothekswesens daraus gezogen werden können; für ein bewusstes »smart« machen.

\section{Einleitung}

Dieses Buch beinhaltet, nahezu vollständig, den Facettenreichtum des RFID-Einsatzes in Bibliotheken. Es werden Entwicklung, Status und zukünftige Trends auf das Tableau gebracht. Darunter sind jedoch einige Beiträge, die es - ungeachtet ihrer inhaltlichen Qualität - nicht geben müsste, wenn die Anfangsjahre bewusster gestaltet worden wären. Es ist nicht ein Phänomen, das nur in Bibliotheken bekannt ist, sondern ein Phänomen der Zeit. Alle Lebensbereiche unterliegen einer beschleunigten Technologisierung, privat, unternehmerisch, eben gesamtgesellschaftlich. Die Zyklen der Erneuerung werden weiterhin kürzer werden. Die fortschreitende Miniaturisierung von Elektronikkomponenten, die positive Preisentwicklung, der Ausbau der Breitbandkommunikationsverbindungen und insbesondere die digitale Konvergenz von Geräten und Diensten sowie die Konvergenz von gewerblichen und privaten Nutzungsprofilen mobiler Endgeräte (Stichwort Bring your own Device) sind nicht mehr nur als Trends auszumachen. Sie sind Fakten einer Mobilen Gesellschaft. Auch das Thema Cloud Computing ist gekoppelt mit der Nutzung mobiler Endgeräte, da durch die Verlagerung von Daten und Software Services die Endgeräte selber noch mehr Applikationen auf kleinstem Raum aufweisen können. Die Entwicklungsgeschwindigkeit von insb. Informations- und Kommunikationstechnologien sind extrem hoch und die digitale Konvergenz führt zur Aufweichung von Systemgrenzen und damit zur Verschmelzung von Inhalten. Dabei geht die informationelle Selbstbestimmtheit durch die Selbstvernetzung der Geräte zunehmend verloren.

Damit berührt das Technologiemanagement als Disziplin immer weitere Kreise. War es zuvor den Technologieexperten überlassen, handeln heute immer mehr Akteure, privat wie beruflich, professioneller im Zusammenhang mit Technologieentscheidungen. Die Debatte um den Datenschutz und die Datensicherheit ist Ausdruck eines Unbehagens gegenüber der Komplexität und in der Sicherheitsforschung wird längst nicht mehr auf das Verdikt der Technologieexperten gesetzt. Dabei wurde deutlich, dass der Versuch, eindimensionale, lineare Kausalketten zu definieren und zu bewerten zunehmend 
scheiterte, die die wissenschaftlichen Einzeldisziplinen disjunkt zu behandeln suchten. Es wurde deutlich, dass der gesellschaftliche Diskurs, die Beteiligung der »Laien « eine Mehrdimensionalität in die Risikobewertung gebracht hat, die der Subjektivität, d. h. der gerechtfertigten, individuellen Wahrnehmung von Risiken, gerecht wurde. [1, S.32 ff.] Vor diesem Hintergrund erhält der Begriff systemische Risiken Einzug in die Diskussion [2, S.21 und die dort zitierte Literatur].

Die Festlegung eines gesellschaftlich tragbaren Restrisikos für einen Technologieeinsatz ist heute politisch nicht mehr legitimiert. Damit steigen aber auch die Verantwortung des Einzelnen und die Notwendigkeit um die komplexen Sachverhalte zu wissen und sie zu bewerten. In Bezug auf den Einsatz von RFID in Bibliotheken ist die Entscheidung über den Einfluss auf den Datenschutz der Akteure selbst zu bewerten und damit auch zu verantworten. Die Europäische Kommission hat dazu ein umfängliches Procedere ${ }^{1}$ geschaffen, das vor dem Hintergrund der Selbstverpflichtung der öffentlichen und privatwirtschaftlichen Nutzung von RFID (und auch NFC) die Folgeabschätzung in die Hände der Akteure legt.

Damit ist der Kompetenzerwerb für das Management von Technologien in Bibliotheken präjudiziert. Dennoch ist nicht alleine die Fragestellung den Schutz der Personen betreffend zu beantworten, um somit die notwendige Akzeptanz für den Erfolg des Einsatzes zu gewährleisten; auch das Verständnis bezogen auf die Vorteilhaftigkeit der Investition muss vorhanden sein. Im Zusammenhang mit dem Technologiemanagement ist nicht nur der Return on Invest (ROI) oder die Amortisationszeit gemeint, sondern auch der Investitionsschutz durch die richtige Wahl einer Technologie. Damit wird mit dem technologiezentrischem Wissensmanagement ein dritter, ohnedies originärer Kompetenzbereich von Bibliotheken adressiert. Woher bekomme ich in Qualität und Aktualität verlässliche Informationen? Zudem soll das gegenseitige Verständnis von Technologieanbietern und Anwendern verbessert werden, um Innovationen zum Vorteil beider Seiten gestalten zu helfen. Dieser Beitrag hat zum Ziel, Handlungsempfehlungen für den Einsatz von neuen Technologien und Konzepten zu geben, wobei RFID Gegenstand und gleichzeitig Präzedenzfall ist.

\section{Zur Genese des RFID-Marktes oder wurde »auf das richtige Pferd gesetzt«}

\section{Vom Technology Push zum Demand Pull}

Ein erklärtes Ziel des Innovationsmanagements [vgl. 3] ist die Zusammenführung von Technology Push und Demand Pull. Das bedeutet, die Anforderungen der Bedarfsträger werden in Form einer Lastenbeschreibung technologieunabhängig formuliert und mit technologischen Entwicklungen im Sinne von Erfindungen abgeglichen. Im Idealfall werden die Entwicklungen darüber erst angestoßen. In der Realität wird jedoch der Prozess umgekehrt, wobei (zufällige) Technologieentwicklungen nach passenden Problemen auf Seiten der Bedarfsträger suchen. Will man diese Zusammenhänge vermitteln, bietet sich die RFID-Technologie als multi-perspektivisches Beispielkonstrukt an. Vieles

1 vgl. Kapitel von Verch, Ulrike: »Datenschutzrechtliche Bedenken beim Einsatz von RFID-Technologie aus europäischer Perspektive zu Privacy Impact Assessment (PIA)« 
von dem was die Theorie von Innovations- und Technologiemanagement zu vermeiden versucht, lässt sich plakativ am Beispiel RFID zeigen.[vgl. 4, S.7-S.43]

Mit der Entwicklung der Smart Label in den frühen 1990ziger Jahren wurde der Grundstein für ein Jahrzehnt exorbitanter »Problemsuche« eingeläutet, die mit dem Start des AutoID Centers in den USA 1999 und nachfolgend der Gründung des epcglobal Konsortiums zu der bekannten Hype-Situation geführt hat. Vor dem Hintergrund einer in weiten Kreisen akzeptierten Henne-Ei Konstellation zwischen Stückpreisanforderungen und eingesetzten Smart Label Mengen wurde auf Seiten der Technologieanbieter vehement in das Geschäftsfeld RFID investiert. Der quartäre Wirtschaftssektor von Konferenzveranstaltern und Marktanalysten heizte mit herausragenden Marktwachstumszahlen das Klima weiter an. Trotz aller Anstrengungen konnten die Implementierungsziele inbs. im Handel nicht erreicht werden. Ab etwa 2007 ist dann interessant zu verfolgen, wie diese Zahlen sukzessive nach unten korrigiert wurden. Investitionen in Geschäftsfelder wurden zurückgefahren oder sie wurden gänzlich geschlossen.

Die Analyse von Technologieadaptionsprozessen wird jährlich seitens der Gartner Group 2 im Rahmen des Gartner Hype Cycles unterstützt. Hierin findet sich neben der Nennung der Technologien auch deren Marktreife. Abbildung 1 zeigt den qualitativen Verlauf und die von Gartner definierten Phasen. Eine Diskussion der RFID-Technologie der Jahre 2005/2006 findet sich z. B. in [4, S. 12].

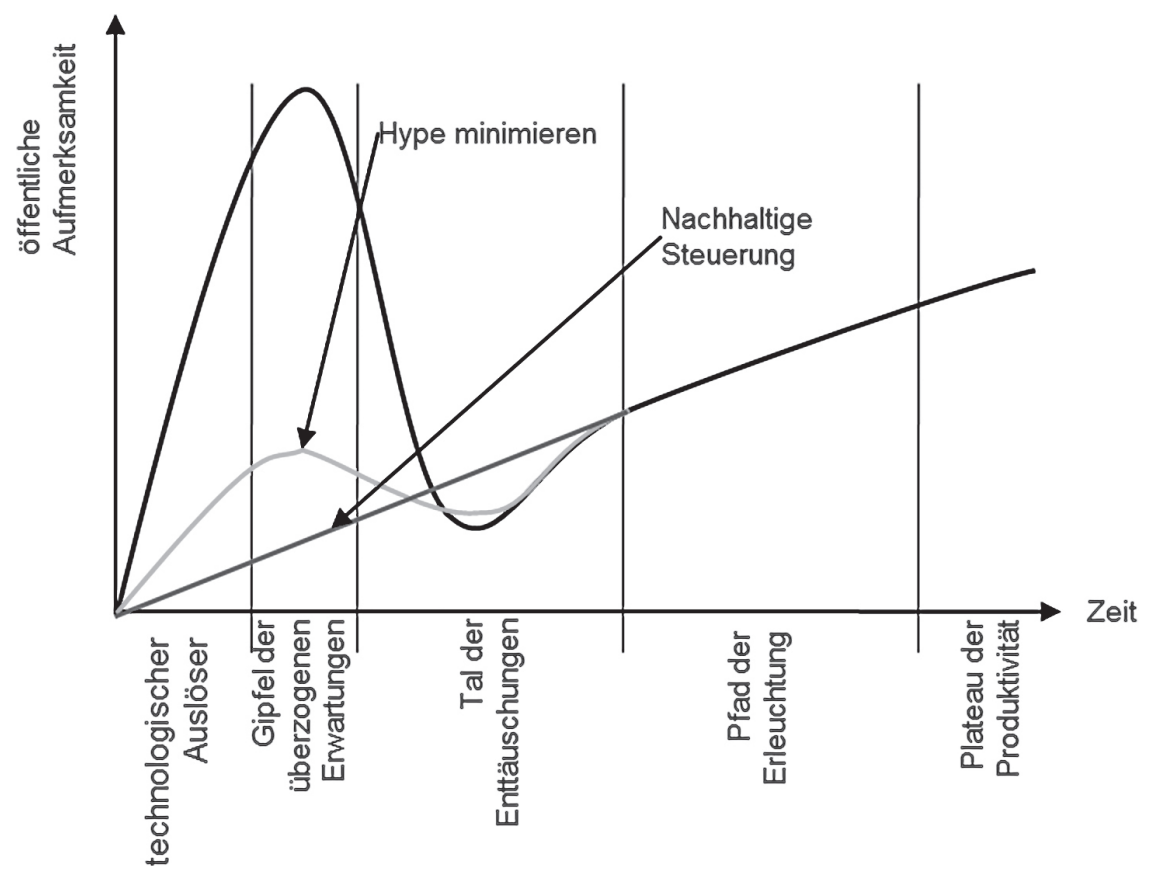

Abbildung 1: Rahmenbedingungen für RFID-Einsatz (in Anlehnung Gartner Hype Cycle ${ }^{3}$ )

2 Gartner Group: http://www.gartner.com

3 ebenda 
Wie in Abbildung 1 dargestellt kann die Einführung von Technologien auch nachhaltig gesteuert werden, so dass eine extreme Ausbildung des »Gipfels überzogener Erwartungen« vermieden wird und damit auch das »Tal der Enttäuschungen« weitaus flacher ist.

Was bedeutet das für den Einsatz von RFID in Bibliotheken. Nachdem die RFID-Industrie in den »Killer-Applikationen « im Handel nicht die gewünschten Wachstumserfolge erzielen konnte, wurde die Suche nach sogenannten »low hanging fruits « gestartet. Das bedeutet, die Komplexitätsbarriere bzw. Kostenbarriere zum Einsatz von RFID ist nicht hoch und es entstehen Referenzprojekte für die Technologie selber oder einfach Umsätze für einen Geschäftsbereich, bzw. Unternehmen. In Abbildung 2 sind die Implementierungsbarrieren dargestellt. Vor dem Hintergrund einer langjährigen Verwendung der Etiketten, verteilen sich die initialen Kosten auf eine Vielzahl von Geschäftsvorgängen und die geschlossene Anwendung innerhalb einer Bibliothek benötigt nicht zwangsläufig mit anderen abgestimmte Standards. ${ }^{4}$

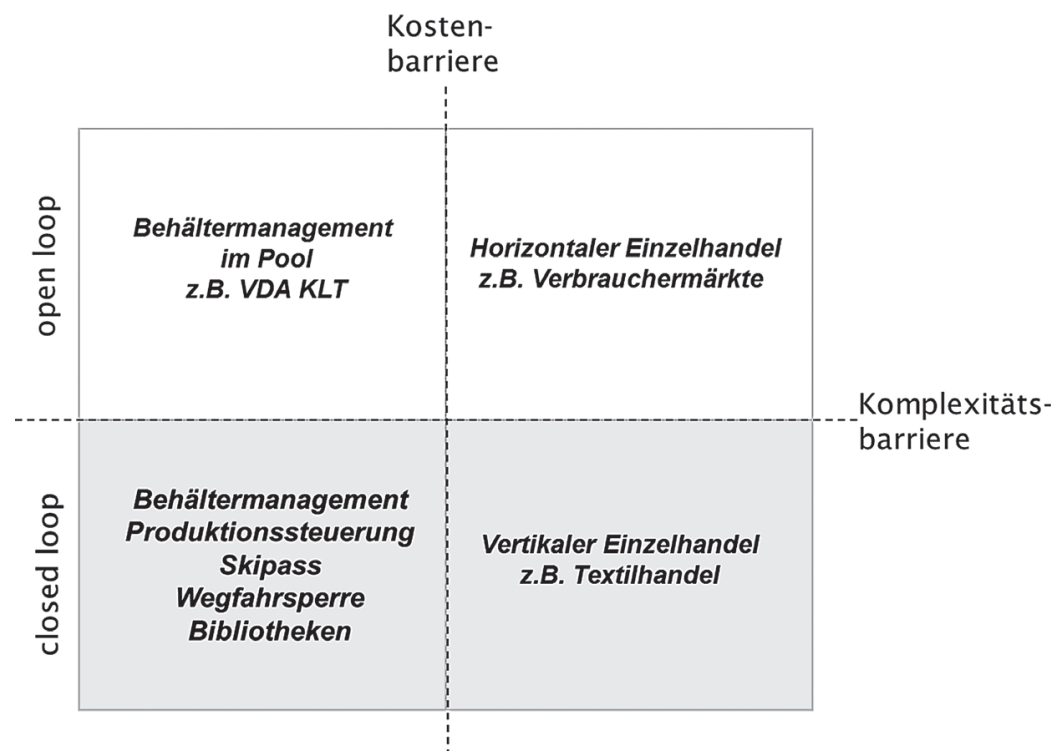

Abbildung 2: Rahmenbedingungen für RFID-Einsatz (in Anlehnung [4, S. 40])

Im Jahr 2001 wurde in Siegburg die erste Bibliothek mit RFID ausgestattet ${ }^{5}$. Dabei wurde auf Basis von im Markt verfügbaren unspezifischen Lesegeräten in Kombination mit einer dafür erstellten Software eine Lösung "gebastelt«. Die Notwendigkeit Leuchtturmprojekte auf Basis RFID zu generieren, führte bereits in den frühen Jahren zu verhältnismäßig niedrigen Etikettenpreisen, in deren Folge Anbieter von Etiketten sich aus dem Geschäft zurückgezogen haben. Die aus Sicht eines Etikettenherstellers geringe Stückzahl pro Fertigungslos hat auf Basis der Zielpreise wenig Raum für Geschäftsmodelle gelassen.

4 das dies dennoch der Fall ist liegt hauptsächlich in den Stückkosten bei großen Mengen gleichartiger Komponenten

5 Der Autor war Projektleiter von Siegburg, Stuttgart und Wien auf der Anbieterseite in den Jahren 2001-2003 
Die Entwicklung der RFID-Technologie ist daher geprägt durch den ständigen Konflikt um Funktionalität und Kosten. Bei sicherheitsrelevanten Applikationen im Bereich der Personenidentifikation überwiegt maßgeblich die Forderung nach Datensicherheit und Datenschutz und führt zu einem hohen Grad an »Security by Design « unter Nutzung komplexer Hardwarelösungen. Im Gegensatz dazu haben sich prozessorientierte Anwendungen wie Bibliotheken im Wesentlichen über die zwei folgenden Faktoren entwickelt:

- Einfachheit der Architektur der RFID Chips zur Kostenminimierung

- Weitreichende offene Standards zur Aktivierung des Economy of Scale und damit Erreichung attraktiver Stückkosten für RFID Tags

Zusätzlich lassen sich Differenzierungsmerkmale durch Qualitätsunterschiede schwer vermitteln. Spezifische Technische Richtlinien, wie sie im industriellen Umfeld die Grundlage von Systemspezifikationen bilden, waren bis zum Jahr 2012 in Bibliotheken nicht vorhanden. Mit der VDI Richtlinie $4478^{6}$ ist dies erstmalig gelungen. Die Richtlinie schlägt die Brücke zwischen den Geschäftsmodellen der Anbieter und den Anforderungen der Bibliotheken im Rahmen der öffentlichen Vergabe, indem Qualitätsunterschiede messbar werden. Dieser Weg sollte weiter beschritten werden. Das bestehende Richtlinienblatt 4478-1 beschreibt RFID Gatesysteme und sollte durch weitere Komponentenbeschreibungen, insb. Etiketten, ergänzt werden. Dies gelingt dann, wenn die Interessenvertreter der Bibliothekare auf der Verbandsseite das Thema institutionalisieren.

Als Fazit kann festgehalten werden, dass RFID-Lösungen in Bibliotheken nicht durch den Einsatz anforderungsgerechter, sondern verfügbarer Komponenten geprägt ist. Unzureichende zukunftsorientierte Anforderungsbeschreibungen lassen weiterhin Raum für Technologieanbieter, Bibliotheken als »Testbed « zu betrachten, anstatt zielorientierte Road-Maps zu entwickeln. Der Status Quo in Bezug auf die Zielpreise hemmen zudem Innovationen, weil bezogen auf die potenzielle Marktgröße aus Sicht von entwicklungsorientierten Unternehmen robuste Geschäftsmodelle nur schwer darzustellen sind.

\section{Die Gestaltung der Zukunft oder Bibliothekare als Innovationsmanager}

Wie eingangs beschrieben, werden die Technologiezyklen kürzer. Die Grundlage ist auch weiterhin das Moore'sche Gesetz, nachdem alle 18 Monate eine Verdopplung der Leistung in der Mikroelektronik erfolgt. Die damit verbundenen Potenziale zum Einsatz innovativer Technologien nehmen damit zu. Diese Potenziale bewirken grundlegende gesellschaftliche Veränderungen, die wiederum Bibliothekskonzepte maßgeblich beeinflussen. Es gilt nun diese Dynamik pro-aktiv zu nutzen, indem Szenarioanalysen mögliche Zukünfte beschreiben helfen. Damit können Anforderungen an den zukünftigen Technologieeinsatz der Zukunft formuliert und Entwicklungsplanungen seitens der Systemanbieter unterstützt werden.

Die Systemanbieter sind naturgemäß gewohnt, Innovationsprozesse professionell zu managen. Je nach Unternehmenskultur nimmt das Innovationsmanagement mehr

6 vgl. Kapitel von Zissel, Gillert: »Qualitätsbestimmung von RFID-Komponenten auf der Basis von allgemein anerkannten Normen und Richtlinien - Vereinfachung von Ausschreibungen« 
oder weniger Raum ein. Ein jedes Unternehmen startet jedoch Entwicklungsprozesse, wenn die Marktchancen gegeben sind und ein Geschäftsmodell vorliegt. Hier gilt es das Verständnis für Geschäftsmodelle seitens der Kunden zu schärfen. Zuweilen ist für Anwender nicht nachzuvollziehen, dass trotz größerer Abnahmemengen, angemessener Preise und ggf. der Übernahme der Entwicklungskosten, dennoch von der Entwicklung Abstand genommen wird. Hier kann der Grund z. B. in anderen Opportunitäten, für die es sich eher lohnt, die Entwicklungskapazitäten zu binden.

Darüber hinaus ist die genaue Beschreibung der Anforderungen derart erforderlich, dass es von Technologienentwicklungsfirmen verstanden wird. Damit sind die spezifische Struktur und der Duktus der Dokumente gemeint.

Der zurzeit existierende Technologieradar ${ }^{7}$ an der ETH Zürich und Fachhochschule Potsdam könnte eine Rolle in der Zukunft spielen, zeigt sich aber eher leblos ${ }^{8}$. Es ist ohnedies davon auszugehen, dass die Institutionalisierung eines technologiestrategischen Kreises eine zentrale, an die Leitung gebundene Struktur benötigt. Zudem müssen die Ziele eines »Think Tanks« klar formuliert werden und im Rahmen von Prozessen abgebildet sein. Die hier angeführten Handlungsempfehlungen können einen Kondensationskeim für die Strukturierung bilden.

\section{Handlungsempfehlungen für einen zentralen Bibliotheks Think Tank:}

1. Willensbekundung und aktive Unterstützung der Leitung der Interessenvertretung von Bibliotheken

2. Szenarienentwicklung für zukünftige Bibliothekskonzepte

3. Zielgerichtetes und kontinuierliches Monitoring von Technologieentwicklungen

4. Kompetenzaufbau für Technologiebewertung und Technologiefolgebewertungen

5. Klassifizierung von Einsatzfeldern/Nutzen

6. Frühzeitige Erstellung von Lastenheften zur Motivation von Innovationen

7. Frühzeitige Erstellung von technischen Beschreibungen (Richtlinien) parallel zu einer Pilotstellung, um die Migration in die Ausschreibungen zu erleichtern

Wenn es gelänge, eine zentrale Anlaufstelle für Technologieanbieter zu schaffen, die einen echten Mehrwert für die Innovationsprozesse bietet, wird aus der Reaktion auf Entwicklungen Aktion und Gestaltungskompetenz.

\section{Fazit}

Seit Beginn des RFID-Einsatzes hat sich viel getan. Die Lösungen sind gereift und funktional ansprechend. Dennoch sind Innovationen in diesem Gebiet weiterhin rar. Dazu müssen Anforderungen klar formuliert und Leistungsunterschiede klar nachgewiesen werden. Damit verbunden, muss auch die Bereitschaft vorhanden sein, die Leistungen angemessen zu honorieren. Sollten dann die Geschäftsmodelle dennoch

7 http://technologieradar.elgg.com/

8 Aufruf Homepage 19.12.2012. Letzter Eintrag Neueste Aktivitäten »12 month ago« 
nicht erfolgreich sein, kann auch dies klar formuliert werden. Im Prozessumfeld (insb. Handel/Industrie) wird zurzeit die UHF-Technologie $\mathrm{e}^{9}$ maßgeblich präferiert, wodurch auch eine Rückkoppelung mit den Bibliotheken entsteht. Gerade im Hinblick auf eine erhoffte Verbesserung der Inventurprozesse. Bezogen auf die in letzten Abschnitt empfohlenen Maßnahmen, sollte eine noch gerade frühzeitige Technologiemanagementbetrachtung erfolgen. Aber RFID ist längst nicht die alleinige Herausforderung; es kommen schnell neue, interessante und nutzenversprechende Technologien - von Ortung und Lokalisierung, über mobile Endgeräte mit Apps, bis zur cloud - für die die Bibliotheken ihre nutzenstiftenden Anforderungen definieren müssen.

\section{Literatur}

[1] Evers, Adalbert, Nowotny, Helga: »Über den Umgang mit Unsicherheit: Die Entdeckung der Gestaltbarkeit der Gesellschaft«, Suhrkamp, Frankfurt am Main, 1987

[2] Renn, Ortwin: »Risiko: Über den gesellschaftlichen Umgang mit Unsicherheit« Oekom Verlag, München, 2007

[3] Hauschildt Jürgen, Salomo, Sören: »Innovationsmanagement«, Verlag Franz Vahlen München, 5. Auflage, 2011

[4] Gillert, Frank, Hansen, Rüdiger: »RFID für die Optimierung von Geschäftsprozessen«, Verlag Hanser München, 2006

9 vgl. Kapitel von Weinländer, Markus, Horst, Dieter: HF oder UHF - Welche Frequenz darf es sein? Vor- und Nachteile der gängigen RFID-Technologien 